\section{Research Square}

Preprints are preliminary reports that have not undergone peer review.

They should not be considered conclusive, used to inform clinical practice, or referenced by the media as validated information.

\title{
Women's Perception of and Readiness to Adopt a Sustainable Healthy Diet: A Cross-sectional Survey in Enugu City, Nigeria
}

Daniel Chukwuemeka Ogbuabor ( $\nabla$ daniel.ogbuabor@unn.edu.ng )

University of Nigeria

Alphonsus Ogbonna Ogbuabor

Enugu State University Teaching Hospital

\section{Research Article}

Keywords: Sustainable diet, healthy diet, dietary behaviour, food choice motives

Posted Date: December 15th, 2021

DOI: https://doi.org/10.21203/rs.3.rs-1046559/v2

License: 우 (i) This work is licensed under a Creative Commons Attribution 4.0 International License. Read Full License 


\section{Abstract}

Background: Perceived benefit of and readiness to adopt sustainable healthy diets (SHDs) is under-investigated in low-resource countries. We assessed women's perceived benefit of and readiness to adopt SHDs and their associated factors in Enugu Metropolis, Nigeria.

Methods: A household cross-sectional survey of childbearing women $(n=450)$ was conducted in January and February 2021 using a questionnaire assessing food choice motives, perceived benefit, and readiness to adopt SHDs. Readiness to adopt SHDs was grouped into pre-contemplation and contemplation $(\mathrm{PC} / \mathrm{C})$, preparation and relapse $(\mathrm{P} / \mathrm{R})$, and action and maintenance $(\mathrm{A} / \mathrm{M})$.

Results: About $79 \%$ and $60 \%$ of women have high perception and adopted SHDs respectively. Perceived benefit of SHD was associated with younger age $(\beta=$ $-0.20, \rho<0.05)$, low education $(\beta=-0.19, \rho<0.05)$, and poor wealth quintile $(\beta=-0.57, \rho<0.001)$. PC/C was predicted by low perceived benefit $(0 R=10.07$, $95 \% \mathrm{Cl}: 4.78-21.22, \rho<0.001)$, low education (OR $=2.51,95 \% \mathrm{Cl}: 1.25-5.04, \rho=0.010)$, and taste $(\mathrm{OR}=3.96,95 \% \mathrm{Cl}: 1.61-9.75, \rho=0.003)$. PR was predicted by low perceived benefit $(\mathrm{OR}=3.92,95 \% \mathrm{Cl}: 1.99-7.73, \rho<0.001)$, low education (OR $=1.82,95 \% \mathrm{Cl}: 1.00-3.29, \rho=0.049)$. A/M was related to younger age $(\mathrm{OR}=$ $0.48,95 \% \mathrm{Cl}: 0.27-0.84, \rho=0.010, \mathrm{PR})$, and health $(\mathrm{OR}=0.14,95 \% \mathrm{Cl}: 0.06-0.36, \rho<0.001, \mathrm{PC} / \mathrm{C})$ and $(\mathrm{OR}=0.17,95 \% \mathrm{Cl}: 0.08-0.35, \rho<0.001, \mathrm{P} / \mathrm{R})$.

Conclusions: Adoption of SHDs need to improve in Enugu, Nigeria. We identified the factors that should inform dietary guidelines and campaigns to increase women's adoption of SHDs.

\section{Introduction}

Achieving a sustainable healthy diet is a growing global concern given the contributions of food choices and consumption behaviours to human and planetary health and implications for achieving the Sustainable Development Goals (SDGs)[1-4]. Sustainable diets are dietary patterns that promote all dimensions of individuals' health; have low environmental pressure and impact; are accessible, affordable, safe, and equitable; and are culturally acceptable[5]. It aims to achieve optimal growth and development of all individuals and support functioning and physical, mental, and social wellbeing at all life stages for present and future generations. Also, it prevents all forms of malnutrition (including undernutrition, micronutrient deficiency, overweight, and obesity), reduce the risk of diet-related non-communicable diseases (NCDs), and supports the preservation of biodiversity and planetary health[5]. Transitioning towards sustainable healthy diets requires substantial population-level changes in food consumption practices. These dietary shifts entail shifting consumption away from animal-based foods and towards more plant-based foods such as fruits, vegetables, nuts, seeds, and whole grains; limiting consumption of highly processed foods and beverages[1, 5, 6]; and balancing nutrient requirements, food costs, and cultural acceptance against environmental impact and other social needs $[1,5,7]$

Unsustainable and unhealthy diets contribute significantly to the risk of NCDs[8, 9]. In low- and middle-income countries, urbanization and increasing prosperity have led to a dietary shift termed nutrition transition in which people consume diets high in calories, hydrogenated fats, sugars, and animal products and low in fibre[10,11]. Highly processed foods that contain high levels of salt, sugar, and fat lead to increasing rates of various chronic diseases, including obesity, diabetes, heart disease, and stroke[12]. Diet-related NCDs are the top risk factors for deaths and disability-adjusted life-years (DALYs) lost globally[13]. In contrast, adherence to a plant-based dietary pattern reduces the risk of diabetes[14, 15$]$. Moreover, adopting a sustainable healthy diet could avert 10.8-11.6 million deaths per year, resulting in $19-24 \%$ of total deaths among adults[1].

The environmental impact of unsustainable and unhealthy diets is also high. Global food systems emit 20-35 per cent of global greenhouse gas (GHG) emissions, occupying about 40 per cent of the Earth's ice-free land area, resulting in terrestrial and aquatic nutrient pollution and biodiversity loss[16]. Overall, the production of animal-based foods has a several-fold higher environmental impact than plant-based foods[17-20]. In the United Kingdom, replacing 50\% of meat and dairy products in the diet with fruits, vegetables, and cereals resulted in a 19\% decrease in GHG emissions[21]. Also, diets that eliminate red meat have a lower global warming potential[22]. Avoiding air-freighted foods, choosing organic over conventional produce, and reducing meat consumption have high environmental benefits $[23,24]$. Nonetheless, consumer awareness of the environmental impact of meat production and willingness to change meat consumption is low $[25,26]$.

Nigeria is one of the few sub-Saharan African countries to develop food-based dietary guidelines (FBDGs)[27]. FBDGs are a set of simple advisory statements that guide consumers on healthy eating patterns, types of food or food groups, or nutrients needed to promote better nutrition and address diet-related health conditions in a country[28]. Notwithstanding Nigeria's FBDGs since 2006, most urban households do not have adequate dietary diversity[29]. Nigeria is experiencing a double burden of malnutrition[30]. About $37 \%, 7 \%, 22 \%$, and $2 \%$ of Nigerian children aged $6-59$ months are stunted, wasted, underweight, and overweight[31]. Also, $12 \%$ of women aged $15-49$ are thin, while $28 \%$ are overweight or obese[31]. Malnutrition was the leading risk factor for death and disability from non-communicable diseases (NCDs) between 2009 and 2019 in Nigeria [32]. The mortality from NCDs increased from $24 \%$ in 2014 to 29\% in $2018[33,34]$. In response to these unacceptable indices, Nigeria's food and nutrition policy aims to attain optimal nutritional status for all Nigerians by addressing the double burden of undernutrition and overweight/obesity[30].

Research on sustainable healthy diets has been conducted mainly in high-income countries and focused on "average diets" extrapolated from national dietary surveys or market data on food, recommended diets by public officials, and hypothetical or optimized versions of diets [1,6,35]. Although most consumers perceived plant-based diets as more beneficial than animal-based diets[36-39], adherence to sustainable dietary behaviours is low [40, 41]. Adoption of SHDs is affected by food choice motives including availability[42], taste[43-45], price[42, 43, 45], health[45-48], weight control[45], and environmental consideration[39, 43,44]. Willingness to adopt a sustainable healthy diet was not associated with socio-demographic characteristics[36, 49]. Conversely, females $[39,49,50]$, young people [39, 51], and high education[42, 48, 51] are more likely to adopt SHDs. 
A significant knowledge gap exists about social and economic aspects of sustainable healthy diets, the drivers of diet, and how scientific information on health and sustainability influence perception and actual practices of consumers[52,53]. The environmental impact and socio-cultural aspects of diet are considered less frequently in national dietary guidelines[4]. In Nigeria, no study has examined the readiness of consumers to adopt sustainable healthy diets. Therefore, this study assessed the perceived benefit of sustainable healthy diets (SHDs), readiness to adopt SHDs, and their associated factors among childbearing women in Enugu State, Nigeria. The findings can inform appropriate policies and strategies to facilitate transitioning to sustainable healthy diets[6].

\section{Materials And Methods Study setting}

The study took place in Enugu metropolis, Enugu State, Nigeria. Enugu metropolis is the capital city of Enugu State and comprises three local government areas (LGAs): Enugu East, Enugu North, and Enugu South LGAs. Whereas Enugu North is composed of an entirely urban population, Enugu East and Enugu North have a mix of urban and rural areas. Enugu East, Enugu North, and Enugu South LGAs comprise 808, 565, and 451 enumeration areas (EAs). In 2020, the estimated population of Enugu metropolis was $1.2 \mathrm{~m}$ people. The publicly owned health facilities in the metropolis include one teaching hospital, three general hospitals, and a network of primary health care facilities.

\section{Research design}

The study adopted a cross-sectional survey design using an interviewer-administered questionnaire.

\section{Study population and sampling strategy}

Childbearing women aged 18 to 49 years living in the Enugu metropolis constituted our study population. We chose childbearing women for this study because mothers make the decisions regarding food and meals in families in Nigeria. A sample size of 344 women was calculated using a single population proportion formula, assuming $70.1 \%$ prevalence for dietary diversity among women in Enugu[31], 95\% confidence limit, $5 \%$ margin of error, and $10 \%$ nonresponse rate. We, however, sampled 450 eligible childbearing using a multi-stage sampling strategy with proportionate size weights. The first stage was to select 20, 14, and 11 enumeration areas from Enugu East, Enugu North, and Enugu South LGAs correspondingly using systematic random sampling. In the second stage, we selected ten households from each enumeration area by systematic sampling. One eligible woman per household was interviewed, totalling 450 respondents.

\section{Data collection}

Data were collected in January 2021 using an interviewer-administered questionnaire. The questionnaire had four sections. Section A covered consumer perception of sustainable dietary habits. We adapted nine questions assessing consumer perception of sustainable healthy diets in a previous study[39]. Women's perceived benefits of sustainable healthy diets were measured using a 5-point Likert scale from 'very small benefit' to 'very large benefit'. In this study, the scale content validity index is 0.95 , while its reliability in our sample is 0.755 .

Section B of the questionnaire measured readiness to adopt sustainable health diets using one question with six response options based on the stages of change construct of the Transtheoretical Model of behaviour change[54]. The response options included: 'I am not interested in doing this at the moment' (precontemplation), 'I am thinking about this but I need more information' (contemplation), 'I would like to do this but there are things stopping me' (planning), 'I have started to do this some of the time' (action), 'I am doing this confidently most of the time' (maintenance), and 'I am not currently doing this but have done in the past' (relapse).

Section $\mathrm{C}$ focused on food choice motives. Childbearing women selected their three most significant food-choice motivations from a provided list (health, cost, religion, taste, environmental sustainability, availability, and animal welfare). Section D covered socioeconomic and demographic information. We measured socioeconomic status using Nigeria's equity tool[55]. Information about age, education level, marital status, and whether participants had children living at home were also collected. Data was collected using an open data kit (ODK) collect (version 1.29.2). Five research assistants, trained to use ODK collect, administered the questionnaire. The interviewers evaluated how the women answered each question and scored the response. The questionnaire was pretested and modified before actual data collection.

\section{Data analysis}

Data were analyzed using SPSS (version 20, IBM, New York, USA). Descriptive statistics were used to summarise the characteristics of respondents, perceived benefit, and food choice motives. The median value of the perceived benefit of sustainable healthy diets was used to dichotomise women into two categories 'low perceived benefit' and 'high perceived benefit'. Also, we reclassified the six stages of change into three categories: pre-contemplation and contemplation $(P C / C)$, preparation and relapse $(P / R)$, action and maintenance $(A / M)$. These three stages reflect groups of individuals who are not interested or may need further information $(P C / C)$, those that experience other barriers $(P / R)$, and those who are already taking action (A/M). ANOVA and t-test were used to test the differences in means perceived benefit of SHD by women's sociodemographic characteristics. We identified determinants of the perceived benefit of SHD using a Generalised linear model. Differences in the proportion of women at different stages of adoption were tested using the Chi-square test. Multinomial 
regression analysis was used to predict women's stage of change for sustainable healthy diets based on perceived benefit, demographic characteristics, and reported food-choice motives. The odds ratio (OR) represents the likelihood of childbearing women being in the PC/C or P/R stages of change compared to the reference, $\mathrm{A} / \mathrm{M}$ stage of change. Statistical significance was set at alpha 0.05 level.

\section{Ethical consideration}

The Health Research Ethics Committee of Enugu State Ministry of Health, Enugu, Nigeria, approved this study. We obtained written, informed consent from all respondents.

\section{Results}

\section{Basic characteristics of the respondents}

The response rate was $100 \%$. All responses were also complete and included in the analysis. Over $50 \%$ of the respondents were in the $25-34$ age group (Table 1). Almost $68 \%$ of respondents were married, about $60 \%$ had tertiary education, and $64 \%$ lived with children. Nearly $83 \%$ of respondents were in the rich quintiles.

Table 1

Socio-demographic characteristics of women of childbearing age, Enugu, Nigeria, 2021

\begin{tabular}{|c|c|c|c|}
\hline Parameters & & Frequency (n) & Percent (\%) \\
\hline \multirow[t]{4}{*}{ Age } & $18-24$ & 99 & 22.0 \\
\hline & $25-34$ & 244 & 54.2 \\
\hline & $35-44$ & 95 & 21.1 \\
\hline & $45-49$ & 12 & 2.7 \\
\hline \multirow[t]{3}{*}{ Marital Status } & Single & 125 & 27.8 \\
\hline & Married & 304 & 67.6 \\
\hline & Other & 21 & 4.7 \\
\hline \multirow[t]{3}{*}{ Education } & Primary & 25 & 5.6 \\
\hline & Secondary/vocational & 157 & 34.9 \\
\hline & Tertiary & 268 & 59.6 \\
\hline \multirow[t]{2}{*}{ Children } & Yes & 287 & 63.8 \\
\hline & No & 163 & 36.2 \\
\hline \multirow[t]{3}{*}{ Socio-economic status } & Poorest/poorer & 37 & 8.2 \\
\hline & Middle & 41 & 9.1 \\
\hline & Richer/Richest & 372 & 82.7 \\
\hline
\end{tabular}

\section{Women's food choice motives}

Health, cost, availability, and taste were the most reported food choice motives among childbearing women (Figure 1).

\section{Perceived benefits of sustainable healthy diets}

Overall, $79 \%$ of women perceived a sustainable healthy diet as a high benefit (Table 2). 'Consume seasonal fruits and vegetables' and 'reduce consumption of air-freighted goods' were perceived to have the highest (84\%) and lowest benefits (54\%), respectively. 
Table 2

Women's perceived benefit of sustainable healthy diets in Enugu, Nigeria, 2021

\begin{tabular}{lllll}
\hline Sustainable health diets & \multicolumn{3}{l}{ Perceived benefit } \\
\cline { 2 - 5 } & Low & \multicolumn{3}{l}{ High } \\
\cline { 2 - 5 } & $\mathrm{n}$ & $\%$ & $\mathrm{n}$ & $\%$ \\
\hline Avoid excess packaging & 164 & 36 & 286 & 64 \\
\hline Buy local produce & 73 & 16 & 377 & 84 \\
\hline Consume fruits and vegetables & 58 & 13 & 392 & 87 \\
\hline Limit red and processed meat & 195 & 43 & 255 & 57 \\
\hline Prioritise plant proteins & 158 & 35 & 292 & 65 \\
\hline Reduce consumption of air freighted foods & 199 & 44 & 251 & 56 \\
\hline Choose sustainable fish & 100 & 22 & 350 & 78 \\
\hline Reduce food waste & 81 & 18 & 369 & 82 \\
\hline Choose organic produce & 114 & 25 & 336 & 75 \\
\hline Overall & 94 & 21 & 356 & 79
\end{tabular}

\section{Factors associated with perceived benefit of sustainable health diets}

As shown in Table 3, the overall perceived benefit of sustainable healthy diets differed significantly by age $(\rho=0.005)$, education $(\rho<0.001)$, and socioeconomic status $(\rho<0.001)$. In all, but two sustainable healthy diets (fruits and vegetables, and sustainable fish), the perceived benefit of sustainable healthy diets differed significantly by education and socioeconomic status. Except for avoiding food with an excessive package, limiting red and processed meat, and eating sustainable fish, all other sustainable healthy diets significantly differed with age. 
Table 3

Women's perceived benefit of sustainable healthy diets disaggregated by socio-demographic characteristics in Enugu, Nigeria, 2021

\begin{tabular}{|c|c|c|c|c|c|c|c|c|c|c|c|c|c|c|c|}
\hline \multirow[t]{3}{*}{ SHDs } & \multicolumn{3}{|l|}{$\mathrm{Age}^{+}$} & \multicolumn{4}{|c|}{ Marital status ${ }^{++}$} & \multicolumn{3}{|c|}{ Education ${ }^{+}$} & \multicolumn{3}{|c|}{ Living with a child ${ }^{+}$} & \multicolumn{2}{|c|}{ Socio-econor } \\
\hline & $<35$ & $35+$ & & Single & Other & Married & & Low & High & & Yes & No & & Poor & Moderat $\epsilon$ \\
\hline & $\mu(S D)$ & $\mu(S D)$ & p-value & $\mu(S D)$ & $\mu(S D)$ & $\mu(S D)$ & $\begin{array}{l}\mathrm{p}- \\
\text { value }\end{array}$ & $\mu(S D)$ & $\mu(S D)$ & p-value & $\mu(S D)$ & $\mu(\mathrm{SD})$ & $\begin{array}{l}\mathrm{p}- \\
\text { value }\end{array}$ & $\mu(S D)$ & $\mu(\mathrm{SD})$ \\
\hline A & $\begin{array}{l}3.7 \\
(1.3)\end{array}$ & $\begin{array}{l}3.9 \\
(1.1)\end{array}$ & 0.068 & $\begin{array}{l}3.8 \\
(1.2)\end{array}$ & $\begin{array}{l}3.9 \\
(1.2)\end{array}$ & $\begin{array}{l}3.7 \\
(1.3)\end{array}$ & 0.639 & $\begin{array}{l}3.5 \\
(1.2)\end{array}$ & $\begin{array}{l}3.9 \\
(1.2)\end{array}$ & $<0.001 *$ & $\begin{array}{l}3.7 \\
(1.3)\end{array}$ & $\begin{array}{l}3.8 \\
(1.2)\end{array}$ & 0.422 & $\begin{array}{l}3.0 \\
(1.3)\end{array}$ & $3.5(1.2)$ \\
\hline B & $\begin{array}{l}4.2 \\
(0.9)\end{array}$ & $\begin{array}{l}4.6 \\
(0.7)\end{array}$ & $<0.001^{*}$ & $\begin{array}{l}4.4 \\
(0.9)\end{array}$ & $\begin{array}{l}4.6 \\
(0.7)\end{array}$ & $\begin{array}{l}4.2 \\
(0.9)\end{array}$ & 0.075 & $\begin{array}{l}4.1 \\
(1.0)\end{array}$ & $\begin{array}{l}4.4 \\
(0.8)\end{array}$ & $0.005^{\star}$ & $\begin{array}{l}4.3 \\
(0.9)\end{array}$ & $\begin{array}{l}4.3 \\
(0.9)\end{array}$ & 0.350 & $\begin{array}{l}3.8 \\
(1.0)\end{array}$ & $4.2(1.0)$ \\
\hline C & $\begin{array}{l}4.3 \\
(0.9)\end{array}$ & $\begin{array}{l}4.5 \\
(0.7)\end{array}$ & $0.034^{*}$ & $\begin{array}{l}4.3 \\
(1.0)\end{array}$ & $\begin{array}{l}4.6 \\
(0.5)\end{array}$ & $\begin{array}{l}4.4 \\
(0.8)\end{array}$ & 0.345 & $\begin{array}{l}4.3 \\
(0.8)\end{array}$ & $\begin{array}{l}4.4 \\
(0.9)\end{array}$ & 0.214 & $\begin{array}{l}4.4 \\
(0.9)\end{array}$ & $\begin{array}{l}4.4 \\
(0.9)\end{array}$ & 0.756 & $\begin{array}{l}4.3 \\
(0.7)\end{array}$ & $4.4(0.9)$ \\
\hline D & $\begin{array}{l}3.6 \\
(1.2)\end{array}$ & $\begin{array}{l}3.8 \\
(1.2)\end{array}$ & 0.182 & $\begin{array}{l}3.6 \\
(1.3)\end{array}$ & $\begin{array}{l}4.1 \\
(0.9)\end{array}$ & $\begin{array}{l}3.6 \\
(1.2)\end{array}$ & 0.137 & $\begin{array}{l}3.3 \\
(1.2)\end{array}$ & $\begin{array}{l}3.8 \\
(1.2)\end{array}$ & $<0.001^{\star}$ & $\begin{array}{l}3.6 \\
(1.2)\end{array}$ & $\begin{array}{l}3.7 \\
(1.2)\end{array}$ & 0.511 & $\begin{array}{l}2.8 \\
(1.4)\end{array}$ & $3.2(1.2)$ \\
\hline$E$ & $\begin{array}{l}3.8 \\
(1.1)\end{array}$ & $\begin{array}{l}4.1 \\
(0.9)\end{array}$ & $0.022^{\star}$ & $\begin{array}{l}3.9 \\
(1.1)\end{array}$ & $\begin{array}{l}3.7 \\
(1.0)\end{array}$ & $\begin{array}{l}3.9 \\
(1.1)\end{array}$ & 0.809 & $\begin{array}{l}3.6 \\
(1.1)\end{array}$ & $\begin{array}{l}4.1 \\
(1.0)\end{array}$ & $<0.001^{\star}$ & $\begin{array}{l}3.8 \\
(1.1)\end{array}$ & $\begin{array}{l}3.9 \\
(1.1)\end{array}$ & 0.451 & $\begin{array}{l}3.2 \\
(1.2)\end{array}$ & $3.5(1.0)$ \\
\hline $\mathrm{F}$ & $\begin{array}{l}3.5 \\
(1.2)\end{array}$ & $\begin{array}{l}3.8 \\
(1.2)\end{array}$ & $0.025^{\star}$ & $\begin{array}{l}3.4 \\
(1.1)\end{array}$ & $\begin{array}{l}3.7 \\
(1.1)\end{array}$ & $\begin{array}{l}3.7 \\
(1.2)\end{array}$ & 0.073 & $\begin{array}{l}3.4 \\
(1.2)\end{array}$ & $\begin{array}{l}3.7 \\
(1.2)\end{array}$ & $0.005^{\star}$ & $\begin{array}{l}3.6 \\
(1.2)\end{array}$ & $\begin{array}{l}3.6 \\
(1.2)\end{array}$ & 0.629 & $\begin{array}{l}2.8 \\
(1.2)\end{array}$ & $3.6(1.1)$ \\
\hline G & $\begin{array}{l}4.1 \\
(1.0)\end{array}$ & $\begin{array}{l}4.2 \\
(1.1)\end{array}$ & 0.642 & $\begin{array}{l}4.0 \\
(1.2)\end{array}$ & $\begin{array}{l}4.6 \\
(0.7)\end{array}$ & $\begin{array}{l}4.2 \\
(1.0)\end{array}$ & $0.048^{*}$ & $\begin{array}{l}4.0 \\
(1.1)\end{array}$ & $\begin{array}{l}4.2 \\
(1.0)\end{array}$ & 0.060 & $\begin{array}{l}4.2 \\
(1.0)\end{array}$ & $\begin{array}{l}4.0 \\
(1.1)\end{array}$ & 0.101 & $\begin{array}{l}4.2 \\
(1.0)\end{array}$ & $4.0(1.1)$ \\
\hline $\mathrm{H}$ & $\begin{array}{l}4.2 \\
(1.1)\end{array}$ & $\begin{array}{l}4.5 \\
(1.0)\end{array}$ & $0.026^{\star}$ & $\begin{array}{l}4.4 \\
(0.9)\end{array}$ & $\begin{array}{l}4.2 \\
(1.2)\end{array}$ & $\begin{array}{l}4.2 \\
(1.1)\end{array}$ & 0.157 & $\begin{array}{l}4.0 \\
(1.2)\end{array}$ & $\begin{array}{l}4.4 \\
(0.9)\end{array}$ & $<0.001^{\star}$ & $\begin{array}{l}4.2 \\
(1.1)\end{array}$ & $\begin{array}{l}4.3 \\
(1.0)\end{array}$ & 0.312 & $\begin{array}{l}3.3 \\
(1.5)\end{array}$ & $4.0(1.3)$ \\
\hline I & $\begin{array}{l}4.0 \\
(1.1)\end{array}$ & $\begin{array}{l}4.3 \\
(0.8)\end{array}$ & $0.009 *$ & $\begin{array}{l}4.0 \\
(1.1)\end{array}$ & $\begin{array}{l}4.2 \\
(0.7)\end{array}$ & $\begin{array}{l}4.1 \\
(1.0)\end{array}$ & 0.373 & $\begin{array}{l}3.9 \\
(1.1)\end{array}$ & $\begin{array}{l}4.2 \\
(1.0)\end{array}$ & $0.005^{\star}$ & $\begin{array}{l}4.1 \\
(1.1)\end{array}$ & $\begin{array}{l}4.2 \\
(1.0)\end{array}$ & 0.367 & $\begin{array}{l}3.2 \\
(1.2)\end{array}$ & $4.0(0.9)$ \\
\hline $\begin{array}{l}\text { Overall } \\
\text { SHD }\end{array}$ & $\begin{array}{l}4.0 \\
(0.7)\end{array}$ & $\begin{array}{l}4.2 \\
(0.6)\end{array}$ & $0.005^{\star}$ & $\begin{array}{l}4.0 \\
(0.6)\end{array}$ & $\begin{array}{l}4.1 \\
(0.6)\end{array}$ & $\begin{array}{l}4.0 \\
(0.7)\end{array}$ & 0.885 & $\begin{array}{l}3.8 \\
(0.8)\end{array}$ & $\begin{array}{l}4.2 \\
(0.6)\end{array}$ & $<0.001^{\star}$ & $\begin{array}{l}4.0 \\
(0.7)\end{array}$ & $\begin{array}{l}4.1 \\
(0.6)\end{array}$ & 0.370 & $\begin{array}{l}3.4 \\
(0.8)\end{array}$ & $3.8(0.8)$ \\
\hline
\end{tabular}

SD = Standard deviation; ${ }^{+}$t-test; ${ }^{++}$ANOVA; ${ }^{*}$ Significant at $<0.05 .(A=$ Avoid excess packaging; $B=$ Buy local produce; $C=$ consume seasonal fruits and vegeta and processed meat; $E$ = Prioritise plant proteins; $F$ = Reduce consumption of air freighted foods; $\mathrm{G}=$ Choose sustainable fish; $\mathrm{H}=\mathrm{Reduce}$ food waste; $\mathrm{I}=\mathrm{Ch}$ produce; mean $=\mu ; \mathrm{SD}=$ standard deviation) .

\section{Predictors of perceived benefits of sustainable healthy diets}

Being poor, age <35 years, and low education significantly predicted the low perceived benefit of sustainable healthy diets (Table 4). 
Table 4

Determinants of perceived benefit of SHD among childbearing women in Enugu, Nigeria, 2021

\begin{tabular}{|c|c|c|c|c|c|c|}
\hline \multirow[t]{2}{*}{ Parameter } & & \multirow[t]{2}{*}{ B } & \multicolumn{2}{|c|}{ 95\% Wald Confidence Interval } & \multicolumn{2}{|l|}{ Hypothesis Test } \\
\hline & & & Lower & Upper & Wald Chi-Square & $\rho$ value \\
\hline & (Intercept) & 4.06 & 3.67 & 4.44 & 426.58 & 0.000 \\
\hline \multirow[t]{2}{*}{ Age (years) } & Age $\leq 35$ & -0.20 & -0.35 & -0.06 & 7.64 & $0.006^{*}$ \\
\hline & Age $>35$ & $0^{\mathrm{a}}$ & & & & \\
\hline \multirow[t]{2}{*}{ Education } & Low education & -0.19 & -0.33 & -0.04 & 6.44 & $0.011^{\star}$ \\
\hline & High education & $0^{\mathrm{a}}$ & & & & \\
\hline \multirow[t]{3}{*}{ Socio-economic status } & Poor & -0.57 & -0.81 & -0.33 & 21.03 & $<0.001^{*}$ \\
\hline & Moderate & -0.21 & -0.43 & 0.01 & 3.35 & 0.067 \\
\hline & Rich & $0^{\mathrm{a}}$ & & & & \\
\hline \multirow[t]{6}{*}{ Food choice motives } & Health & 0.16 & -0.03 & 0.35 & 2.70 & 0.101 \\
\hline & Cost & 0.03 & -0.14 & 0.19 & 0.09 & 0.762 \\
\hline & Taste & 0.05 & -0.11 & 0.22 & 0.40 & 0.528 \\
\hline & Environ & 0.06 & -0.17 & 0.29 & 0.26 & 0.611 \\
\hline & Availability & 0.15 & -0.02 & 0.31 & 3.13 & 0.077 \\
\hline & (Scale) & $0.43^{b}$ & 0.37 & 0.49 & & \\
\hline
\end{tabular}

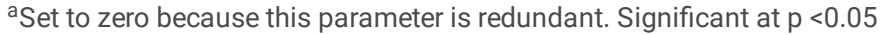

b.Maximum likelihood estimate.

\section{Factors associated with adoption of sustainable healthy diets}

About $19 \%, 21 \%$, and $60 \%$ of women are pre-contemplation and contemplation (PC/C); preparation and relapse (P/R); and action and maintenance (A/M) correspondingly. Adoption of SHDs significantly differed in some socio-demographic factors except in 'marital status' and 'leaving with a child at home'. Also, the adoption of sustainable healthy diets differed significantly by food choice motives except for the environment (Table 5). As women transit from PC/C through $P / R$ to $A / M$, the proportion of women with the perceived high benefit of sustainable healthy diets significantly increases $(\rho<0.001)(T a b l e ~ 5)$. 
Table 5

Readiness to adopt sustainable healthy diets disaggregated by women's characteristics in Enugu, Nigeria, 2021

\begin{tabular}{|c|c|c|c|c|c|c|c|c|}
\hline \multirow[t]{3}{*}{ Parameters } & & \multicolumn{7}{|c|}{ Readiness to adopt Sustainable healthy diet } \\
\hline & & \multicolumn{2}{|c|}{$\mathrm{PC} / \mathrm{C}(\mathrm{n}=86)$} & \multicolumn{2}{|c|}{$P / R(n=96)$} & \multicolumn{2}{|c|}{$\mathrm{A} / \mathrm{M}(\mathrm{n}=268)$} & \multirow[t]{2}{*}{$\mathrm{P}$ value } \\
\hline & & $\mathrm{n}$ & $\%$ & $\mathrm{n}$ & $\%$ & $\mathrm{n}$ & $\%$ & \\
\hline \multirow[t]{2}{*}{ Age (years) } & $<35$ & 78 & 90.7 & 61 & 63.5 & 204 & 76.1 & $<0.001 *$ \\
\hline & $\geq 35$ & 8 & 9.0 & 35 & 36.0 & 64 & 24.0 & \\
\hline \multirow[t]{2}{*}{ Education } & Low & 56 & 65.1 & 50 & 52.1 & 76 & 28.4 & $<0.001^{*}$ \\
\hline & High & 30 & 34.9 & 46 & 47.9 & 192 & 71.6 & \\
\hline \multirow[t]{3}{*}{ Marital status } & Single & 26 & 30.2 & 23 & 24.0 & 76 & 76.1 & 0.806 \\
\hline & Others & 3 & 3.5 & 6 & 37.0 & 12 & 25.0 & \\
\hline & Married & 57 & 66.3 & 67 & 69.8 & 180 & 67.2 & \\
\hline \multirow[t]{2}{*}{ Leaving with a child at home } & Yes & 54 & 62.8 & 66 & 68.8 & 167 & 62.3 & 0.519 \\
\hline & No & 32 & 37.2 & 30 & 31.3 & 101 & 76.1 & \\
\hline \multirow[t]{3}{*}{ Socio-economic status } & Poor & 14 & 90.7 & 13 & 38.0 & 10 & 26.0 & $<0.001 *$ \\
\hline & Moderate & 9 & 10.0 & 11 & 11.5 & 21 & 7.8 & \\
\hline & Rich & 63 & 73.3 & 72 & 75.0 & 237 & 88.4 & \\
\hline \multirow[t]{2}{*}{ Health } & No & 53 & 61.6 & 35 & 36.5 & 27 & 76.1 & $<0.001^{\star}$ \\
\hline & Yes & 33 & 38.4 & 61 & 39.0 & 241 & 27.0 & \\
\hline \multirow[t]{2}{*}{ Cost } & No & 15 & 17.4 & 22 & 22.9 & 99 & 36.9 & 0.001 \\
\hline & Yes & 71 & 82.6 & 74 & 77.1 & 169 & 63.1 & \\
\hline \multirow[t]{2}{*}{ Taste } & No & 16 & 18.6 & 42 & 43.8 & 132 & 76.1 & $<0.001 *$ \\
\hline & Yes & 70 & 81.4 & 54 & 40.0 & 136 & 28.0 & \\
\hline \multirow[t]{2}{*}{ Environment } & No & 82 & 90.7 & 86 & 89.6 & 234 & 87.3 & 0.110 \\
\hline & Yes & 4 & 11.0 & 10 & 10.4 & 34 & 12.7 & \\
\hline \multirow[t]{2}{*}{ Availability } & No & 20 & 23.3 & 36 & 37.5 & 128 & 76.1 & $<0.001^{*}$ \\
\hline & Yes & 66 & 76.7 & 60 & 41.0 & 140 & 29.0 & \\
\hline \multirow[t]{2}{*}{ Perceived benefit of SHD } & Low & 43 & 50.0 & 27 & 28.1 & 24 & 9.0 & $<0.001^{*}$ \\
\hline & High & 43 & 50.0 & 69 & 71.9 & 244 & 91.0 & \\
\hline
\end{tabular}

Significant at $\mathrm{p}<0.05$

\section{Predictors of adoption of sustainable healthy diets}

Health predicted women's likelihood of being in the $A / M$ stage of change for sustainable healthy diets compared to $P C / C(O R=0.14, \rho<0.001)$, and $P / R(O R=$ $0.17, \rho<0.001)$ stages of change (Table 6$)$. Low perceived benefit of sustainable healthy diets predicted being in the $P C / C(O R=10.07, \rho<0.001)$ and $P / R$ $(\mathrm{OR}=3.92, \rho<0.001)$ stages of change, correspondingly. Taste, as a food choice motive, was significantly associated with being in the PC/C stage of change $(O R=3.96, \rho=0.003)$. While low education was associated with being in the $P C / C$ stage $(O R=2.51, \rho=0.010)$ and $P / R$ stage $(O R=1.82, \rho=0.049)$ compared to $A / M$ stage. Age $<35$ years predicted the likelihood of women being in the $A / M$ stage compared to $P / R(O R=0.48, \rho=0.010)$ stage of change. 
Table 6

Predictors of readiness to adopt sustainable healthy diets among women in Enugu State, Nigeria, 2021

\begin{tabular}{|c|c|c|c|c|c|c|c|c|c|c|c|}
\hline \multicolumn{2}{|c|}{ Readiness to adopt SHDa } & \multicolumn{5}{|l|}{$\mathrm{PC} / \mathrm{C}$} & \multicolumn{5}{|l|}{$P / R$} \\
\hline & & \multirow[t]{2}{*}{ B } & \multirow[t]{2}{*}{ Sig. } & \multirow[t]{2}{*}{$\mathrm{OR}$} & \multicolumn{2}{|c|}{$\begin{array}{l}95 \% \text { Confidence Interval for } \\
\text { OR }\end{array}$} & \multirow[t]{2}{*}{ B } & \multirow[t]{2}{*}{ Sig. } & \multirow[t]{2}{*}{ OR } & \multicolumn{2}{|c|}{$\begin{array}{l}\text { 95\% Confidence Interval for } \\
\text { OR }\end{array}$} \\
\hline \multicolumn{2}{|l|}{ Predictors } & & & & Lower Bound & Upper Bound & & & & Lower Bound & Upper Bound \\
\hline \multicolumn{2}{|l|}{ Intercept } & -3.32 & 0.001 & & & & .37 & .586 & & & \\
\hline \multirow[t]{4}{*}{ Food motives } & Health & -1.95 & $<0.001^{*}$ & 0.14 & 0.06 & 0.36 & -1.80 & $<0.001^{\star}$ & 0.17 & 0.08 & 0.35 \\
\hline & Cost & 0.59 & 0.161 & 1.80 & 0.79 & 4.09 & 0.17 & 0.600 & 1.18 & 0.63 & 2.20 \\
\hline & Taste & 1.38 & $0.003^{*}$ & 3.96 & 1.61 & 9.75 & 0.00 & 0.994 & 1.00 & 0.53 & 1.90 \\
\hline & Availability & 0.83 & 0.064 & 2.30 & 0.95 & 5.54 & -0.20 & 0.558 & 0.82 & 0.42 & 1.60 \\
\hline \multirow{2}{*}{$\begin{array}{l}\text { Perceived } \\
\text { benefit }\end{array}$} & Low & 2.31 & $<0.001^{\star}$ & 10.07 & 4.78 & 21.22 & 1.37 & $<0.001$ * & 3.92 & 1.99 & 7.73 \\
\hline & High & $0^{\mathrm{b}}$ & & & & & $0^{\mathrm{b}}$ & & & & \\
\hline \multirow{3}{*}{$\begin{array}{l}\text { Socio- } \\
\text { economic }\end{array}$} & Poor & 0.33 & 0.588 & 1.39 & 0.42 & 4.59 & 0.82 & 0.123 & 2.26 & 0.80 & 6.40 \\
\hline & Moderate & -0.61 & 0.283 & 0.54 & 0.18 & 1.66 & -0.06 & 0.890 & 0.94 & 0.37 & 2.35 \\
\hline & Rich & $0^{\mathrm{b}}$ & & & & & $0^{\mathrm{b}}$ & & & & \\
\hline \multirow[t]{2}{*}{ Age (years) } & $<35$ & 0.78 & 0.094 & 2.19 & 0.88 & 5.46 & -0.74 & 0.010 * & 0.48 & 0.27 & 0.84 \\
\hline & $\geq 35$ & $0^{\mathrm{b}}$ & & & & & $0^{\mathrm{b}}$ & & & & \\
\hline \multirow[t]{2}{*}{ Education } & Low & 0.92 & $0.010^{*}$ & 2.51 & 1.25 & 5.04 & 0.60 & $0.049 *$ & 1.82 & 1.00 & 3.29 \\
\hline & High & $0^{\mathrm{b}}$ & & & & & $0^{\mathrm{b}}$ & & & & \\
\hline
\end{tabular}

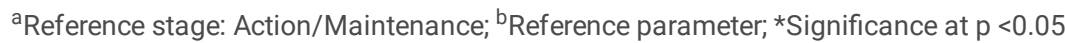

\section{Discussion}

This study has examined women's perceived importance of and readiness to adopt sustainable healthy diets and the factors associated with perceived benefits and adoption of sustainable healthy diets (SHDs) in a Nigerian urban city. The findings revealed that most women considered SHDs of high benefit, but adoption was moderate. Perceived benefit, food motives (health and taste), and socio-demographic factors (age and education) predicted the adoption of SHDs. This evidence provides pointers to strategies to facilitate transitioning to sustainable healthy diets.

Our finding of the high perceived benefit of SHDs among childbearing women is consistent with the evidence of previous studies[23, 37-39, 45, 56]. This finding might reflect an increasing awareness of diet-related NCDs, health consciousness and high nutrition value of SHDs, and renewed interest in traditional foods among Nigerians[57]. Nevertheless, our sample differs from previous existing evidence in some ways. For instance, while 'consuming seasonal fruits and vegetables' conferred the highest benefit in our study, 'avoiding air-freighted goods' conferred the highest benefit in a previous study [39]. Also, our findings that 'limiting red and processed meat' and 'reduce consumption of air-freighted goods' offered the lowest benefits contrast results from a previous study in which 'prioritise plant protein' and 'choose organic produce' were perceived to confer the smallest benefits [39]. These variations in the perceived benefits of sustainable healthy diets are context-specific and should inform strategies intended to improve the adoption of SHDs among women.

Despite the high perceived benefit of SHDs among women in our sample, adoption is moderately high, which aligns with findings from Europe[45], but differs from the evidence of low willingness to adopt SHDs[25, 40,41]. Consistent with the Transtheoretical Model of behaviour change[54], we found that adoption of SHDs increased with its perceived benefit. This finding aligns with the results of previous studies[39, 45, 50, 58]. In this study, women with low perceived benefits were about ten and four times more likely to be in the PC/C and P/R stage of change correspondingly. Generally, as women transit from PC/C through $P / R$ to $A / M$, the proportion of women with low perceived benefit declines, while the proportion of women with high benefit increases. Yet, the women's high perceived benefit did not always translate to $\mathrm{A} / \mathrm{M}$ as some women with high perception were in the $\mathrm{PC} / \mathrm{C}$ and $\mathrm{P} / \mathrm{R}$ stages. Therefore, interventions to improve the adoption of SHDs must target two sub-populations - women with low perceived benefit and those with high perceived benefit who are in $\mathrm{PC} / \mathrm{C}$ and $\mathrm{P} / \mathrm{R}$.

Health predicted the likelihood of women being in the A/M stage of change for all sustainable healthy diets, thus, confirming our hypothesis that health would predict readiness to adopt SHDs. Health concerns increased women's adoption of sustainable healthy diets as was found in prior studies[39, 45, 46]. This finding is not surprising because, in this sample, health was the foremost food choice motive, implying that health and nutrition benefits of foods take precedence over all other motivations for food choice. Evidence indicates that health as a food choice motive positively correlates with better adherence to healthy nutritional guidelines[48]. While health was also the leading food choice motive in the United Kingdom and Tanzania, it was the fourth-ranked motivation for food choice in Europe[39, 45, 58]. In European settings where health is not a foremost food choice motive, people believe that they already eat a 
healthy diet and therefore do not consider SHDs would provide benefits over and above their existing dietary behaviour. Health consciousness resulting from increasing NCDs may have influenced consumers to adopt SHDs[57, 58].

Our finding that women reporting taste as a food choice motive were more likely to be in the PC/C stage confirmed our hypothesis that taste would predict low readiness to adopt SHDs. These findings are similar to the results of previous studies where concern for taste was associated with less healthy dietary habits[44, 45]. Conversely, a previous study found that taste influenced the adoption of plant-based proteins but no other SHDs[39]. As argued elsewhere[45], the assumption that other food choice motives would be considered before taste when selecting sustainable healthy diets may have adversely influenced the readiness to adopt SHDs. In our sample, reporting taste as the fourth important motivation for food choice supports this assumption.

We hypothesized that increased age would have a high perceived benefit and more likely to adopt SHDs. However, while increased age was associated with higher perceived benefits, it did not translate to the adoption of SHDs. Our finding that younger women were more likely to be in the A/M stage contrasts evidence of a prior study in which younger consumers were more likely to be PC/C stage for SHDs[39]. Our findings are surprising from three perspectives.

First, in a traditional food system, people prefer locally produced foods, legumes, seasonal vegetables, and fruits to animal source foods and highly processed foods[3]. However, young women in Nigeria tend to eat few traditional foods and prefer imported and western goods, perceived as appealing and healthy[59]. Secondly, young women tend to be vulnerable to media influences that promote air-freighted foods[59]. Thirdly, consumption of sugary foods decreased with age among Nigerian women[31]. One possible explanation of our finding is the large proportion of younger women in our sample who reported health concerns as a food choice motive. Given that attitudes relate to individuals and others and the intention to act is personalised[45], it might also be that younger women are more proactive than older women in translating their perceived benefit into action. There is a need to target older women with behaviour change communication interventions.

This study's findings confirmed our hypothesis that high education was associated with high perceived benefits and high readiness to adopt SHDs. Existing evidence suggests that high education predicts the adoption of SHDs[42, 48, 51]. Although socioeconomic status was not a significant predictor of readiness to adopt SHDs in this study, differences in education might reflect income disparities in urban Nigeria. Women in low-income urban households have lower educational qualifications, poorer intake of micronutrients, and less diversified diets than high-income urban households in Nigeria [29]. Education increases the opportunities for employment, which improves financial access to diverse, sustainable healthy diets [29, 59]. Targeting information campaigns on maternal nutrition and health knowledge towards women with a lower level of education might improve their adoption of SHDs.

This study contributes to existing scholarship by providing evidence of perceived benefit and motivation to adopt sustainable healthy diets in Nigeria. This evidence can inform the adaptation of FBDGs to improve women's access to SHDs in urban areas of South-East Nigeria. These notwithstanding, social desirability bias might limit the study. Women in this study may have projected a socially desirable image of food choice motives and dietary behaviours. The use of an interviewer-administered questionnaire reduced the chances of response biases. Secondly, the perceived benefit of SHDs may not have adequately captured environmental benefits. In this study, environmental concern was the fifth important motivation for food choice. Evidence elsewhere suggests that the impact of food on the environment is poorly understood, and consumers have not yet internalised environmental sustainability[51]. Therefore, the interpretation of the environmental effects of food in Nigeria constitutes an area for future studies. Equally, it would be a helpful addition to exploring the adoption of sustainable dietary behaviour from a qualitative perspective.

\section{Conclusions}

This study has highlighted the gap between perceived benefit and readiness to adopt sustainable healthy diets and the factors associated with the adoption of SHDs in a South-Eastern Nigerian City. High perceived benefit does not always translate to the adoption of SHDs. Health concern, taste, younger age, and high formal education are critical determinants of readiness to adopt SHDs and might be considered in interventions to accelerate the transition to sustainable healthy diets.

\section{Abbreviations}

A/M: Action and Maintenance; DALY: Disability-adjusted life-years; EAs: Enumeration areas; FBDG: Food-based dietary guidelines; GHG: Global greenhouse gas; LGAs: Local government areas; NCDs: Non-communicable diseases; ODK: Open data kit; PC/C: Precontemplation/Contemplation; P/R: Preparation and Relapse; SDGs: Sustainable development goals; SHD: sustainable healthy diets; UK: United Kingdom;

\section{Declarations}

\section{Ethics approval and consent to participate}

The study was conducted according to the guidelines of the Declaration of Helsinki and approved by the Institutional Review Board (or Ethics Committee) of Enugu State Ministry of Health (protocol code MH/MSD/REC21/163 and 6 January 2021). Written, informed consent was obtained from all subjects involved in the study.

\section{Consent for publication:}

Not applicable 


\section{Availability of data:}

The data presented in this study are available on request from the first author

\section{Competing Interests:}

The authors report no conflict of interests.

\section{Funding:}

The authors received no external funding for this study.

\section{Author Contributions:}

Conceptualization and literature review, D.O. and A.O.; methodology, D.O. and A.O; data analysis, D.O.; writing-original draft preparation, D.O.; writing-review and editing intellectual content, D.O. and A.O.; finalisation of article, D.O. All authors have read and agreed to the published version of the manuscript.

\section{Acknowledgements:}

We acknowledge the National Population Commission Enugu State Office for assisting us in selecting the enumeration areas in the study area; and Uche Ezema for developing the open data kit (ODK) questionnaires used in this study.

\section{Authors information:}

Ogbuabor Daniel, MBBS, PhD - Orcid No: 0000-0002-9617-1538. Daniel is a Senior Lecturer, Department of Health Administration and Management, University of Nigeria, Enugu Campus, Nigeria.

Alphonsus Ogbuabor, PhD, is a Senior Scientist at the Enugu State University Teaching Hospital, Parklane, Enugu, Nigeria.

\section{References}

1. Willett W, Rockström J, Loken B, Springmann M, Lang T, Vermeulen S, Garnett T, Tilman D, DeClerck F, Wood A, et al: Food in the Anthropocene: the EATLancet Commission on healthy diets from sustainable food systems. Lancet 2019, 393:447-492.

2. Sievert K, Lawrence M, Parker C, Baker P: Understanding the Political Challenge of Red and Processed Meat Reduction for Healthy and Sustainable Food Systems: A Narrative Review of the Literature. Int J Health Policy Manag 2020.

3. Fanzo J: Healthy and Sustainable Diets and Food Systems: the Key to Achieving Sustainable Development Goal 2? Food Ethics 2019, 4:159-174.

4. Martini D, Tucci M, Bradfield J, Di Giorgio A, Marino M, Bo D, \#039, C, Porrini M, Riso P: Principles of Sustainable Healthy Diets in Worldwide Dietary Guidelines: Efforts So Far and Future Perspectives. Nutrients 2021, 13:1827.

5. FAOandWHO: Sustainable healthy diets - Guiding principles. Rome: Food and Agricultural Organisation and World Health Organisation; 2019.

6. Springmann M, Wiebe K, Mason-D'Croz D, Sulser TB, Rayner M, Scarborough P: Health and nutritional aspects of sustainable diet strategies and their association with environmental impacts: A global modelling analysis with country-level detail.. Lancet Planet Health 2018, 2:e451-e461.

7. Drewnowski A, Finley J, Hess JM, Ingram J, Miller G, Peters C: Towards healthy diets from sustainable food system. Curr Dev Nutr $2020,4: \mathrm{nzaa083.}$

8. McClements DJ: Future foods: Is it possible to design a healthier and more sustainable food supply? Nutr Bulletin 2020, 45:341-354.

9. Branca F, Lartey A, Oenema S, Aguayo V, Stordalen GA, Richardson R, Arvelo M, Afshin A: Transforming the food system to fight non-communicable diseases. Brit Med J 2019, 364:I29.

10. Ruini LF, Ciati R, Pratesi CA, Marino M, Principato L, Vannuzzi E: Working toward Healthy and Sustainable Diets: The "Double Pyramid Model" Developed by the Barilla Center for Food and Nutrition to Raise Awareness about the Environmental and Nutritional Impact of Foods. Front Nutr 2015, 2:9.

11. Popkin BM: Relationship between shifts in food system dynamics and acceleration of the global nutrition transition. Nutr Rev 2017, 75:73-82.

12. Hall KD, Ayuketah A, Brychta R, Cai H, Cassimatis T, Chen KY, Chung ST, Costa E, Courville A, Darcey V, et al: Ultra-Processed Diets Cause Excess Calorie Intake and Weight Gain: An Inpatient Randomized Controlled Trial of Ad Libitum Food Intake. Cell Metab 2019, 30:67-77.e63.

13. Afshin A, Sur PJ, Fay KA, Cornaby L, Ferrara G, Salama JS, Mullany EC, Abate KH, Abbafati C, Abebe Z, Afarideh M: Health effects of dietary risks in 195 countries, 1990-2017: a systematic analysis for the Global Burden of Disease Study 2017. Lancet 2019, 393:1958-1972.

14. Qian, Liu G, Hu FB, Bhupathiraju SN, Sun Q: Association Between Plant-Based Dietary Patterns and Risk of Type 2 Diabetes: A Systematic Review and Meta-analysis. JAMA Intern Med 2019, 179:1335-1344.

15. Lee Y, Park K: Adherence to a Vegetarian Diet and Diabetes Risk: A Systematic Review and Meta-Analysis of Observational Studies. Nutrients 2017, 9:603.

16. IPCC: Global warming of $1.5^{\circ} \mathrm{C}$ : an IPCC special report on the impacts of global warming of $1.5^{\circ} \mathrm{C}$ above pre-industrial levels and related global greenhouse gas emission pathways, in the context of strengthening the global response to the threat of climate change, sustainable development, and 
efforts to eradicate poverty.: Intergovernmental Panel on Climate Change; 2018.

17. Lonnie M, Johnstone AM: The public health rationale for promoting plant protein as an important part of a sustainable and healthy diet. Nutr Bull 2020 , 45:281-293.

18. Poore J, Nemecek T: Reducing food's environmental impacts through producers and consumers. Science 2018, 360:987-992.

19. Aleksandrowicz L, Green R, Joy EJM, Smith P, Haines A: The impacts of dietary change on greenhouse gas emissions, land use, water use, and health: $A$ systematic review. PLoS One 2016, 11:e0165797.

20. Nelson ME, Hamm MW, Hu FB, Abrams SA, Griffin TS: Alignment of healthy dietary patterns and environmental sustainability: a systematic review. Adv Nutr 2016, 7:1005-1025.

21. Scarborough P, Allender S, Clarke D, Wickramasinghe K, Rayner M: Modelling the health impact of environmentally sustainable dietary scenarios in the UK. Eur J Clin Nutr 2012, 66:710-715.

22. Veeramani A, Dias G, Kirkpatrick S: Carbon footprint of dietary patterns in Ontario, Canada: a case study based on actual food consumption. $J$ Clean Prod 2017, 162.

23. Jungbluth N, Tietje O, Scholz RW: Food purchases: impacts from the consumers' point of view investigated with a modular LCA. Int $J$ Life Cycle Assess 2000, 5:134-142.

24. BDA: One blue dot: the BDA's ground-breaking project for sustainable diets.: British Dietary Association; 2018.

25. Hartmann C, Siegrist M: Consumer perception and behaviour regarding sustainable protein consumption: A systematic review. Trends Food Sci Tech $2017,61: 11-25$.

26. Macdiarmid JI, Douglas F, Campbell J: Eating like there's no tomorrow: Public awareness of the environmental impact of food and reluctance to eat less meat as part of a sustainable diet. Appetite 2016, 96:487-493.

27. Herforth A, Arimond M, Álvarez-Sánchez C, Coates J, Christianson K, Muehlhoff E: A Global Review of Food-Based Dietary Guidelines. Adv Nutr 2019, 10:590-605.

28. Hailu Bekele TH, de Vries JJHM, Trijsburg L, Feskens E, Covic N, Kennedy G, Brouwer ID: Methodology for developing and evaluating food-based dietary guidelines and a Healthy Eating Index for Ethiopia: a study protocol. BMJ Open 2019, 9:e027846.

29. Obayelu OA, Osho FR: How diverse are the diets of low-income urban households in Nigeria? J Agric Food Res 2020, 2:100018.

30. FMBNP: National policy on food and nutrition in Nigeria. 2016. Abuja, Nigeria: Federal Ministry of Budget and National Planning; 2016.

31. NPC[Nigeria] and ICF: Nigeria demographic and health survey 2018. Abuja, Nigeria, and Rockville, Maryland, USA: National Population Commission and ICF; 2019.

32. GBD 2019 Risk Factors Collaborators: Global burden of 87 risk factors in 204 countries and territories, 1990-2019: a systematic analysis for the Global Burden of Disease Study 2019. Global Health Metrics 2020, 396:P1223-1249.

33. WHO: Noncommunicable diseases country profiles 2018. Geneva, Switzerland: World Health Organisation; 2018.

34. WHO: Noncommunicable diseases country profile 2014. Geneva, Switzerland: World Health Organisation; 2014.

35. Chen C, Chaudhary A, Mathys A: Dietary Change Scenarios and Implications for Environmental, Nutrition, Human Health and Economic Dimensions of Food Sustainability. Nutrients 2019, 11:856.

36. Lea EJ, Crawford D, Worsley A: Consumers' readiness to eat a plant-based diet. Eur J Clin Nutr 2006, 60:342-351.

37. Reipurth M, Hørby L, Gregersen C, Bonke A, Perez CFJ: Barriers and facilitators towards adopting a more plant-based diet in a sample of danish consumers.. Food Qual Prefer 2019, 73:288-292.

38. Vanhonacker F, Van Loo EJ, Gellynck X, Verbeke W: Flemish consumer attitudes towards more sustainable food choices. Appetite 2013, $62: 7-16$.

39. Culliford A, Bradbury J: A cross-sectional survey of the readiness of consumers to adopt an environmentally sustainable diet. Nutr $\mathrm{J} 2020,19: 138$.

40. Nocella G, Srinivasan CS: Adherence to WHO's nutrition recommendations in the UK: dietary patterns and policy implications from a national survey. Food Policy 2019, 86:101719.

41. Yau A, Adams JM, Monsivais P: Time trends in adherence to UK dietary recommendations and associated sociodemographic inequalities, 1986-2012: A repeated cross-sectional analysis. Eur J Clin Nutr 2019, 73:997-1005.

42. Fink L, Strassner C, Ploeger A: Exploring External Factors Affecting the Intention-Behavior Gap When Trying to Adopt a Sustainable Diet: A Think-Aloud Study. Front Nutr 2021, 8:511412.

43. Wekeza SV, Sibanda M: Factors Influencing Consumer Purchase Intentions of Organically Grown Products in Shelly Centre, Port Shepstone, South Africa. Int J Environ Res Public Health 2019, 16:956.

44. Allès B, Péneau S, Kesse-Guyot E, Baudry J, Hercberg S, Méjean C: Food choice motives including sustainability during purchasing are associated with a healthy dietary pattern in french adults. Nutr $\mathrm{J} 2017,16: 58$.

45. Rankin A, Bunting BP, Poínhos R, van der Lans IA, Fischer AR, Kuznesof S, Almeida M, Markovina J, Frewer LJ, Stewart-Knox BJ: Food choice motives, attitude towards and intention to adopt personalised nutrition. Public Health Nutr 2018, 21:2606-2616.

46. Shrestha A, Baral S: Consumers' willingness to pay for organic agriculture products: a case study of Nepalgunj city, Banke. Int J Agric Environ Food Sci 2019, 3:58-61.

47. Wang X, Pacho F, Liu J, Kajungiro R: Factors Influencing Organic Food Purchase Intention in Developing Countries and the Moderating Role of Knowledge. Sustainability 2019, 11:209. 
48. Lê J, Dallongeville J, Wagner A, Arveiler D, Haas B, Cottel D, Simon C, Dauchet L: Attitudes toward healthy eating: a mediator of the educational level-diet relationship. Eur J Clin Nutr 2013, 67:808-814.

49. Tobler C, Visschers VHM, Siegrist M: Eating green. Consumers' willingness to adopt ecological food consumption behaviours. Appetite 2011, 57:674-682.

50. de Boer J, de Witt A, Aiking H: Help the climate, change your diet: A cross-sectional study on how to involve consumers in a transition to a low-carbon society. Appetite 2016, 98:19-27.

51. Sánchez-Bravo P, Chambers E, Noguera-Artiaga L, López-Lluch D, Chambers E, Carbonell-Barrachina ÁA, Sendra E: Consumers' Attitude towards the Sustainability of Different Food Categories. Foods 2020, 9:1608.

52. Ernstoff A, Stylianou KS, Sahakian M, Godin L, Dauriat A, Humbert S, Erkman S, Jolliet O: Towards Win-Win Policies for Healthy and Sustainable Diets in Switzerland. Nutrients 2020, 12.

53. Vermeir I, Weijters B, De Houwer J, Geuens M, Slabbinck H, Spruyt A, Van Kerckhove A, Van Lippevelde W, De Steur H, Verbeke W: Environmentally Sustainable Food Consumption: A Review and Research Agenda From a Goal-Directed Perspective. Front Psycho/ 2020, 11:1603.

54. Prochaska J, Velicer W: The trans-theoretical model of health behaviour change. Am J Health Promot 1997, 12:38-48.

55. Metrics for Management: Nigeria equity tool. vol. 2018: Metric for Measurement; 2015.

56. Hoolohan C, Berners-Lee M, McKinstry-West $\mathrm{J}$, Hewitt CN: Mitigating the greenhouse gas emissions embodied in food through realistic consumer choices. Energy Policy 2013, 63:1065-1074.

57. Maziya-Dixon B, Achterbosch T, Adelekan D, Adeyemi O, Ajieroh V, Akerele D, Akinola A, Alamu E, van Berkum S, Byrd K, et al: Food systems for healthier diets in Nigeria: A research agenda Washington, DC: International Food Policy Research Institute (IFPRI); 2021.

58. Pacho F: What influences consumers to purchase organic food in developing countries? Brit Food J 2020, 122:3695-3709.

59. Mapis GJ: The Dietary Decision-Making Process of Women in Nigeria. Doctoral. Walden University College of Health Sciences; 2020.

\section{Figures}

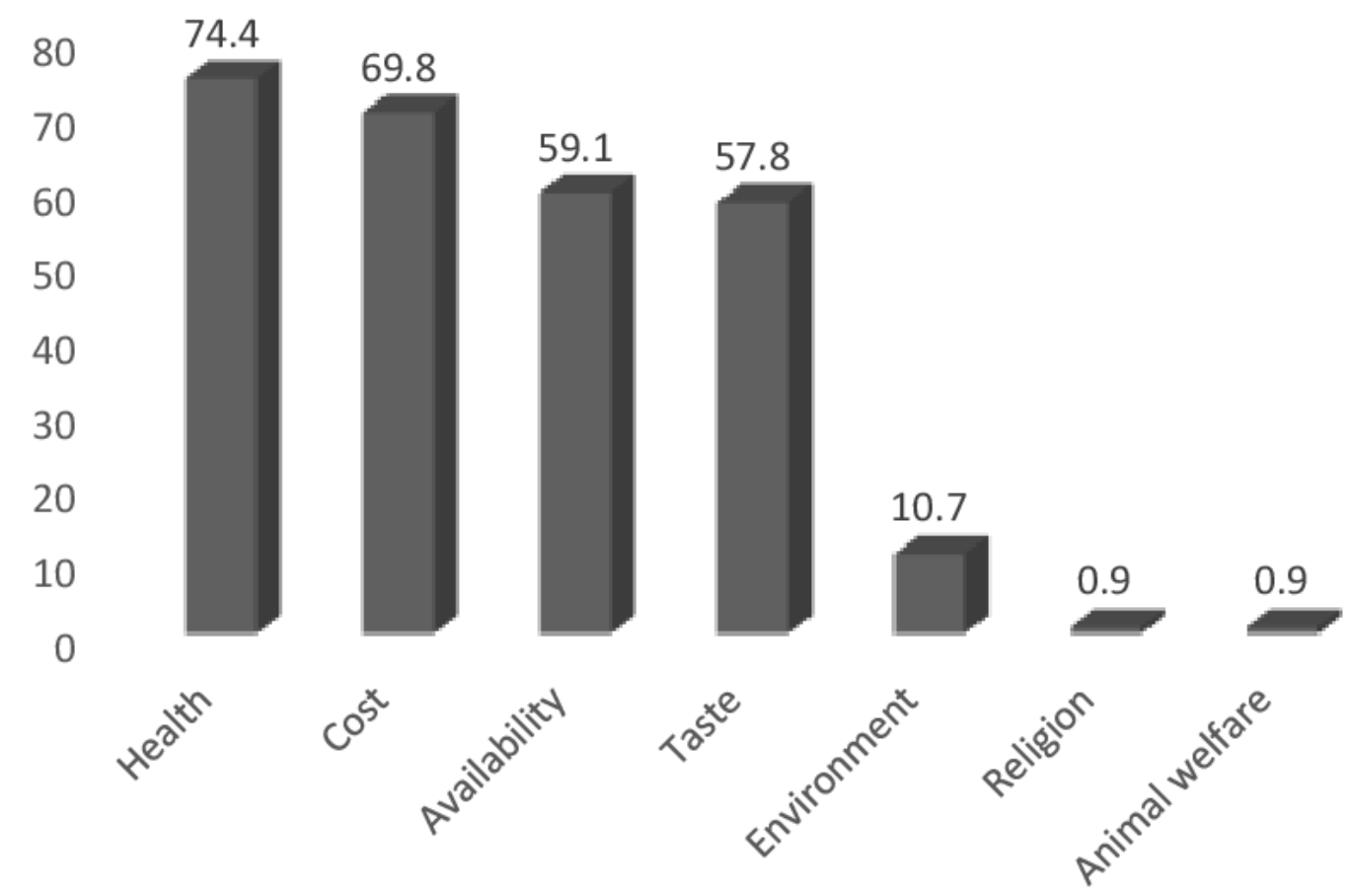

Figure 1

Motivations for food choices among childbearing women in Enugu, Nigeria, 2021 